\title{
Evaluation of the limits of resonance tunability in metallic nanoshells with a spectral averaging method
}

\author{
Stefan Schelm and Geoff B. Smith \\ Department of Applied Physics, University of Technology, Sydney, P.O, Box 123 Broadway, \\ 2007 New South Wales, Australia
}

Received January 7, 2005; accepted January 19, 2005

Spectral selectivity based on tuning the surface plasmon resonance in metallic nanoshells by variation of the relative shell thickness is shown to be limited by the interplay between scattering and absorption. To achieve resonance energies in the near infrared and infrared, relatively large cores are needed, which lead to strong and broad scattering bands and multipolar contributions in the visible. The scattering contribution to extinction is described with a new parameter $\mathcal{S}_{\lambda}$, which is defined for a wavelength range of interest $\Lambda$. This parameter can help in designing materials for specific applications where scattering is either hindering, as in nearinfrared absorbers for visually clear windows, or actually desired, such as in particle-array-based sensors. (C) 2005 Optical Society of America

OCIS codes: $290.0290,290.4020$.

\section{INTRODUCTION}

Metallic nanoparticles and metal nanoshells have attracted a lot of interest in recent years, both in basic research ${ }^{1-5}$ and for applications. ${ }^{6-8}$ This interest is based to a large degree on the plasmon resonance properties of these systems. Especially for optical applications, though, there remain some unanswered questions regarding the effect of scattering. Most approaches in this area consider structures that are small compared with the wavelength of the light and hence employ quasi-static limits. The validity of the approximation has to be considered carefully, especially for systems consisting of many nanostructures and where clustering or aggregation can occur.

The onset of scattering for a given system is a strong indication that quasi-static limits are not applicable anymore. A systematic understanding of the conditions when scattering becomes noticeable is therefore important in the understanding of limits in the use of quasi-static approaches. This discussion also applies, for example, to the area of composite materials, where effective medium approximations are a powerful method for describing optical properties. This is because effective medium approximations are also based on quasi-static limits.

Another important effect of scattering is its effect on the tunability of the resonance and, related to this, on the spectral selectivity of nanoscale systems. We demonstrate this effect with the example of metallic nanoshells.

One of the main interests in core-shell structures is the desire to achieve spectral selectivity, that is, a distinct contrast between extended neighboring bandwidths in the optical properties. This is achieved in nanoshells by tuning the spectral position of the surface plasmon resonance by changing the ratio of the shell thickness to the core radius. As this tuning depends on the relative shell thickness, there remains the question of whether certain ratios can be achieved experimentally before the particles become too large and scattering destroys spectral selectivity. One of the most important parameters in determining scattering is the overall size of the particle, and it is thus expected that the tuning range is reduced when scattering becomes noticeable or even dominant. This can present an inherent design limit to the ability to localize the resonance in metal nanoshells.

The importance of the contribution of scattering toward extinction is not confined to the testing of the design limits for metallic shell absorption. Scattering at nonvisible wavelengths, for example, can be tolerated and can even improve the efficiency of certain applications. Further important points are the wavelength range and the suspending medium, as they differ between applications and have an influence on the scattering. Particles suspended in water, for example, scatter generally more at visible wavelengths than if they are suspended in polymers. Our proposed approach is able to address all of the points mentioned above by using a new spectrally averaged scattering and absorption parameter. This property has features distinct from other monochromatic parameters.

\section{THEORY}

What is desired is a general approach that can be used for all materials, sizes, and shapes, to measure the amount of scattering evident in extinction for a certain wavelength range. The property usually used to quantify the amount of scattering is the albedo, $W_{0}=C_{\text {sca }} / C_{\text {ext }}$, where $C_{\text {sca }}$ is the scattering cross section and $C_{\text {ext }}$ is the extinction cross section. To acquire a quality factor for a given wavelength range, one could simply sum or integrate the albedo, but this would introduce the wavelength as a unit into the quality factor, which should be dimensionless.

We propose to determine the scattering contribution by defining the ratio $\mathcal{S}_{\mathrm{A}}$ between the amount of energy that 
is scattered, $W_{\text {sca }}$, to the amount that is totally extinct, $W_{\text {ext }}$, from the incident beam in the wavelength region $\Lambda$ :

$$
\mathcal{S}_{1}=\frac{\int_{1} W_{\text {sca }} \mathrm{d} \lambda}{\int_{\Lambda} W_{\text {ext }} \mathrm{d} \lambda}
$$

If we use $W_{\text {sca,ext }}=C_{\text {sca,ext }} I_{i}$, where $I_{i}$ is the incident irradiance, $\mathcal{S}_{\mathrm{A}}$ can be rewritten in terms of the cross sections or efficiencies $Q_{\text {sca,ext }}=C_{\text {sca,ext }} / G, G$ being the geometric cross section, as

$$
\mathcal{S}_{\Lambda}=\frac{\int_{\lambda} C_{\mathrm{sca}}(\lambda) I_{i}(\lambda) \mathrm{d} \lambda}{\int_{\Lambda} C_{\mathrm{ext}}(\lambda) I_{i}(\lambda) \mathrm{d} \lambda}=\frac{\int_{\Lambda} Q_{\mathrm{sca}}(\lambda) I_{i}(\lambda) \mathrm{d} \lambda}{\int_{\Lambda} Q_{\mathrm{ext}}(\lambda) I_{i}(\lambda) \mathrm{d} \lambda}
$$

With this equation we can determine the desired quantity $\mathcal{S}_{\mathrm{I}}$ from efficiency spectra calculated for a given scatterer and wavelength range. Equation (2) does not depend on how the spectra were obtained and can therefore be applied to any situation in which the spectra can be calculated, e.g., for spherical or nonspheroidal particles or for single particle or clusters, or can be measured. We stress at this point that the proposed approach yields a value different from the above-mentioned simple summation or integration of the albedo, which is evident from comparing the definition of the albedo and Eq. (2). We believe that the value $\mathcal{S}_{\Lambda}$ does express the desired situation in a better way than solutions based on the albedo directly.

We used a public Mie code developed by Bohren and Huffman $^{9}$ to calculate the full Mie absorption and scattering efficiencies for homogeneous gold and silver spheres and dielectric-core metallic-shell particles. The Drude part of the dielectric function for the metals was size corrected by use of the following equation ${ }^{10}$.

$$
\epsilon(R)=\epsilon_{\mathrm{bulk}}+\omega_{p}^{2}\left[\frac{1}{\omega^{2}+i \omega \gamma_{x}}-\frac{1}{\omega^{2}+i \omega \gamma(R)}\right],
$$

where $\epsilon_{\text {bulk }}$ is the bulk dielectric function of the metal (gold from Ref. 11 and silver from Ref. 12), $\omega_{p}$ the bulk plasma frequency, $\gamma_{x}$ the inverse of the bulk relaxation time, and $\left.\gamma(R)=\gamma_{\times}+A v_{F} / R\right)$ a size-dependent relaxation frequency, where $R$ is the radius of the particle, $v_{F}$ is the Fermi velocity of the metal, and $A$ is a parameter that in principle depends on the theory considered in the particle surface scattering process ${ }^{13}$ but that can also be used to empirically describe additional broadening mechanisms $^{14,15}$ (e.g., defects, grain boundaries, and chemical interface effects). To provide results closer to experimental realities we used $A=2$, which creates an effective mean free path of half the physical size of the particle. This is a good approximation for some systems ${ }^{14,16-18}$ and seems even a lower limit for other core-shell systems. ${ }^{4}$

For the effective radius used in Eq. (3) for the shells, we chose a model proposed by Granqvist and Hunderi ${ }^{19}$ :

$$
R_{\text {eff }}=\frac{1}{2}\left[\left(r_{\text {shell }}-r_{\text {core }}\right)\left(r_{\text {shell }}^{2}-r_{\text {core }}^{2}\right)\right]^{1 / 3},
$$

where $R_{\text {eff }}$ is the effective mean free path used in the determination of $\gamma(R)$ and $r$ is the radius of the core or the shell, respectively.

The use of $\mathcal{S}_{1}$ is not limited to the case studied here but should be able to improve material design whenever either the scattering or the absorbtion contribution to extinction is of importance. For this purpose $Q_{\text {sca }}$ can be exchanged with $Q_{a b s}$ in Eq. (2), $Q_{a b s}$ being the absorption efficiency. What might also be of interest in certain cases, such as color pigments, could be relations based on Eq. (2) with both $Q_{\text {sca }}$ and $Q_{\text {abs }}$ present instead of $Q_{\text {ext }}$.

\section{RESULTS AND DISCUSSION}

We calculated extinction, scattering, and absorption efficiencies for solid gold and silver particles as well as the respective metallic-shell $\mathrm{SiO}_{2}$-core particles in a medium with $n_{\text {host }}=1.49$. All of the calculations were done with the appropriate size correction from Eq. (3) and the effective radius of Eq. (4) for the core-shell particles. In the calculation of the scattering contribution $\mathcal{S}_{1}$ from Eq. (2), the irradiance $I_{i}$ was assumed to be constant over the wavelength range considered here.

Figure 1 shows a three-dimensional plot of $\mathcal{S}_{\Lambda}$ for the gold core-shell systems studied and the wavelength range $\Lambda$ of $300-2000 \mathrm{~nm}$. The plane shows the results for the different core-shell particles, and the results for the homogeneous spheres are shown as circles, for which we fix

$$
\delta=\frac{\text { shell thickness }}{\text { core radius }}=1 .
$$

The position within the $x-y$ plane defines the total radius $r_{\text {total }}=r_{\text {core }}+t_{\text {shell }}$ of the particle. What can immediately be seen, as expected, is that the largest particles have the largest scattering contribution. The results for $\mathcal{S}_{\Lambda}$ are also smaller in the coated spheres than in the solid particles, independent of $\delta$ which indicates that the metallic shells are generally producing less scattering than the homogeneous spheres.

This is shown more clearly in Fig. 2, where the contribution $S_{\Lambda}$ is shown as a function of the total radius of the

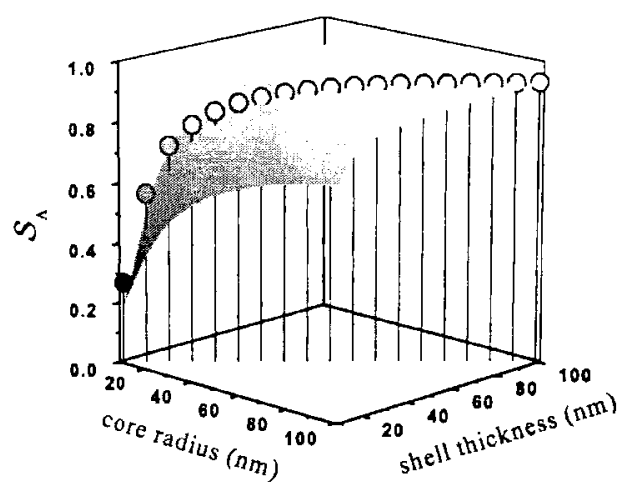

Fig. 1. Three-dimensional plot of the scattering contribution $S_{\text {, }}$ for the studied gold-shell $\mathrm{SiO}_{2}$-core particles in a medium with $n_{\text {host }}=1.49$. The circles represent results for solid gold particles (shown with an arbitrary $\delta$ ratio of 1 ). 


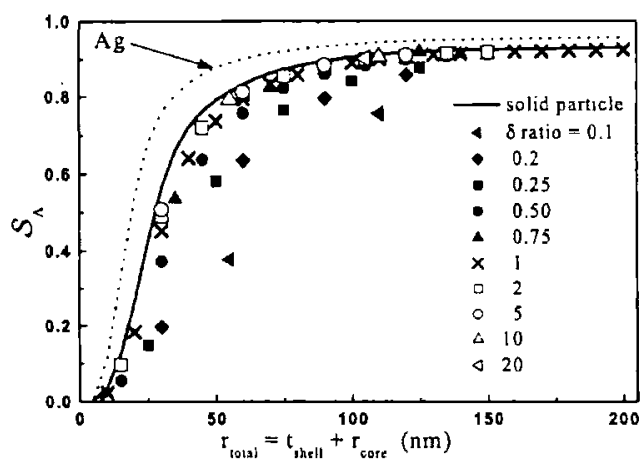

Fig. 2. Scattering contribution for gold nanoshells for the wavelength range $300-2000 \mathrm{~nm}$ as a function of the total radius of the particle for different $\delta$ ratios in a medium with $n_{\text {host }}=1.49$. Solid curve, homogeneous gold spheres; dotted curve, homogeneous silver spheres.

scatterer for the homogeneous particle and different $\delta$ ratios of the gold shell. Again, the wavelength range $A$ is $300-2000 \mathrm{~nm}$. It can be seen that the values of $\mathcal{S}_{1}$ for the coated system are always smaller than those for the homogeneous spheres. This is true even for $\delta$ ratios of up to 20 . The results for $\delta=2$ are, on the other hand, already close to the results for the solid sphere at small total radii. This is due to the efficient shielding of the core by the shell.

Results for silver shells are very similar; therefore we show only the result for solid spheres of silver in Fig. 2 as a comparison. Please note that each of the points in this graph represents the results from one set of data, i.e., results from two spectra. This succinct representation is one of the main advantages of the proposed approach.

The reason that $\mathcal{S}_{1}$ does not reach the expected value of 1 , even for the largest spheres, is the occurrence of interband absorption below $600 \mathrm{~nm}$ for gold. This means that, despite the stronger Rayleigh scattering for these shorter wavelengths, the bound electron excitations are strong enough to quench the scattering. This can be seen as a crossing of the solid $Q_{\mathrm{sca}}$ and $Q_{\mathrm{abs}}$ below $600 \mathrm{~nm}$ in Fig. 3 . Despite the stronger overall scattering for this larger sphere, $Q_{\text {abs }}$ becomes larger than $Q_{\text {sca }}$ in regions where interband transitions are occurring. This causes the specific value of the asymptotic limit to be a function of the material as well as the studied wavelength range.

The effect of a different scattering contribution on efficiency spectra can be seen in Fig. 3. It shows scattering, absorption, and extinction efficiencies for gold shells with $r_{\text {total }}=110 \mathrm{~nm}$ and $r_{\text {total }}=55 \mathrm{~nm}$ for the same ratio $\delta=0.1$. These two sets of $\delta=0.1$ curves in Fig. 3 lead to the two $\delta=0.1$ points at total radii of $110 \mathrm{~nm}$ and $55 \mathrm{~nm}$ plotted in Fig. 2. The relatively stronger scattering, $S_{1}=0.76 \mathrm{com}$ pared with $\mathcal{S}_{1}=0.38$ for the smaller particle, as well as the multipole contributions for the larger particle can be clearly observed. If one considers a maximum value for $\mathcal{S}_{\wedge}$ of 0.4 , to keep the scattering low, the maximum resonance position for a gold-on-silica shell with experimentally achievable shell thicknesses $(\approx 5 \mathrm{~nm})$ seems to be 1100 $\mathrm{nm}$, as seen in Fig. 3. This is well short of the possible tuning range shown in Fig. 1(b) of Ref. 2 (up to $10 \mu \mathrm{m}$ ).

Nonetheless, near-infrared energies seem to be attainable with experimentally possible systems, which is an in- teresting region for applications in its own right, but a further shift into the infrared without the onset of scattering in the visible seems impossible. $\mathrm{Au} / \mathrm{Au}_{2} \mathrm{~S}$ systems, on the other hand, might be more flexible at smaller sizes, as the gold shell is created out of the core material, and thinner shells are possible. ${ }^{20,21}$

As can be seen from Fig. 2, the only way to reduce $\mathcal{S}_{A}$ and thereby retain the tunability in the absorption for larger particles (around $r_{\text {total }}=100 \mathrm{~nm}$ ) is to use very thin metallic layers with ratios $\delta \approx 0.1$. This requires a metallic layer with a thickness of only $10 \mathrm{~nm}$ for a particle of $r_{\text {total }}=100 \mathrm{~nm}$ and even smaller shells for smaller total sizes. To experimentally achieve a smooth, continuous metallic shell of this thickness is challenging, and a discontinuous, poor-quality shell would decrease the electron mean free path $R_{\text {eff }}$ even further. This increases the broadening and decreases the strength of the resonance, in effect decreasing the effectiveness of the shells. Another question is whether the size correction, as described in Eqs. (3) and (4) is still valid for extremely thin shells.

Experimental values from the literature for $\delta$ and $r_{\text {total }}$ for gold shells on silica cores are in the range $\delta=0.106$ to $\delta=0.550$ and $r_{\text {total }}=80$ to $r_{\text {total }}=293 \mathrm{~nm},{ }^{2,4,6,7}$ and for gold shells on $\mathrm{Au}_{2} \mathrm{~S} \delta=0.130$ to $\delta=0.270$ for $r_{\text {total }}=5.1$ to $r_{\text {total }}$ $=20.7 \mathrm{~nm}{ }^{20,21}$ The smallest ratio for the silica core particles of $\delta=0.106$ was achieved for a core radius of $170 \mathrm{~nm}$ and $r_{\text {total }}=188 \mathrm{~nm}$. $^{2}$ This is well within the regime where the scattering contribution is at its maximum and spectral tuning of the resonance with the relative shell thickness is no longer possible.

To highlight the reduced tuning capabilities for larger particles, we include Fig. 4, where we show the extinction efficiencies for gold-shell-on-silica-core spherical particles for a total radius of $r_{\text {total }}=150 \mathrm{~nm}$ and for $\delta$ between 0.5 and 2 , as well as a larger core-shell spheres, with $r_{\text {total }}$ $=200 \mathrm{~nm}$ and $\delta=1$, for comparison. It is almost impossible to see any differences in the spectra for the $150 \mathrm{~nm}$ particles, independent of the actual value of $\delta$. For these sizes, where scattering dominates the extinction, the ratio of the shell thickness to the core radius cannot be used to tune the spectral response. Only a change in the total size of the particle changes the spectra slightly. Even then, the position and shape of the two main resonances at $600 \mathrm{~nm}$ remain almost unchanged, and only the broader and

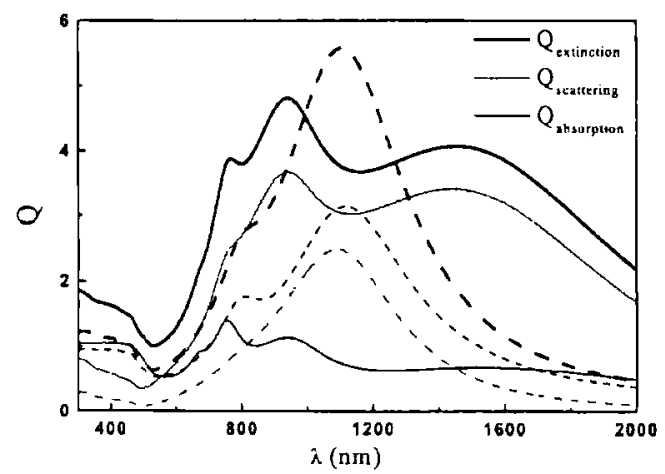

Fig. 3. Extinction, scattering, and absorption efficiencies for two different spherical gold shells on a silica core, both with $\delta=0.1$ but with different total radii: $r_{\text {total }}=110 \mathrm{~nm}$ (solid curves) and $r_{\text {total }}=55 \mathrm{~nm}$ (dashed curves) 


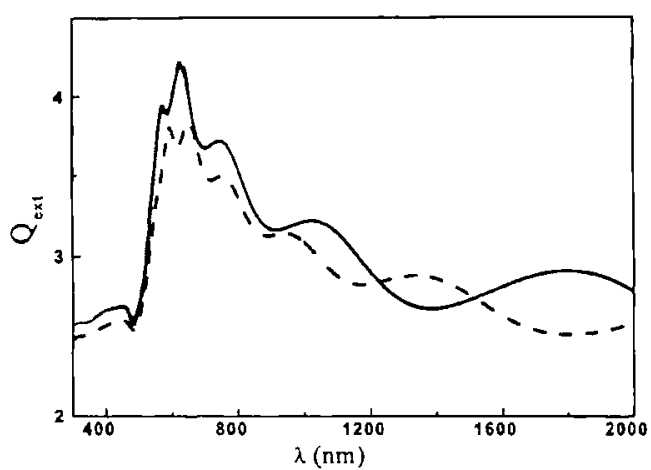

Fig. 4. Extinction efficiencies for spherical gold nanoshells with a silica core. Solid curves, $r_{\text {total }}=150 \mathrm{~nm}$ and $\delta$ between 0.5 and 2 (curves are actually so close that they are indistinguishable). Dashed curve, $r_{\text {total }}=200 \mathrm{~nm}$ and $\delta=1$.

weaker, lower-order multipole peaks at longer wavelengths are shifted. Hence this scattering-dominated size range shows almost no effect of $\delta$ on the spectral tunability, compared with sizes for which absorption is dominant.

We point out that we only presented examples of the efficiency spectra to highlight the advantages of the scattering contribution $\mathcal{S}_{\Lambda}$. What we showed here is that with the help of these examples one can estimate the general form of the efficiency spectra, with respect to the strength of the scattering, for other systems based on the $\mathcal{S}_{1}$ value alone. This proposed figure of merit could improve rational material design, where scattering is involved or needs to be checked, by removing the need to study multiple spectra and condensing the desired information into a single value.

\section{SUMMARY AND CONCLUSION}

We showed that there is a limit to spectral selectivity and tunability of the surface plasmon resonance of metallicshell dielectric-core particles and that this limit is reached surprisingly quickly at $r_{\text {total }} \gtrless 125 \mathrm{~nm}$. This is an inherent design limit, as a large tuning range requires small ratios of shell thickness to core radius. This, in turn, requires either high-quality, extremely thin shells $(<5 \mathrm{~nm}$, for particles smaller than $50 \mathrm{~nm})$, which are very difficult to achieve experimentally, or larger particles to keep the thickness-to-core ratio constant with thicker and experimentally better achievable, high-quality shells. The disadvantage in the latter case is that the larger particles will scatter significantly and thereby reduce the absorption and diminish the tuning capability.

While this information can be deduced from standard cross sections and efficiencies, we presented a more directly accessible way to quantify both spectral selectivity and the scattering contribution to extinction with the introduction of the scattering contribution $\mathcal{S}_{1}$. The ratio $\mathcal{S}_{1}$ permits the comparison of the scattering contribution for different materials, sizes, and shapes for a specific wavelength range and subsequent quality assessment of each system. Depending on the desired properties of the system of interest, other ratios of scattering, absorption, and extinction, as in Eq. (2), can be derived, resulting in other quality parameters like $\mathcal{S}_{1}$.
One might argue that a spectrally averaged value actually hides the spectral selectivity and that hence $\mathcal{S}_{A}$ is of little use for this problem. Nonetheless, $\mathcal{S}_{1}$ is a sufficient and concise parameter to evaluate spectral selectivity. The reason for this is that, as long as the the spectral selectivity is achieved by absorption, an increase of scattering diminishes the relative contribution of absorption and by that reduces spectral selectivity. This follows from the fact that extinction bands due to scattering are broader (owing to the usual onset of multipole contributions in scattering) than those due to absorbtion.

Furthermore, a good understanding of the scattering properties of a certain system is necessary in order to assess the validity of quasi-static approximations, which also has an effect on quasi-static based methods like effective medium approximations. It also allows one to estimate the effect of scattering on the tunability of resonances and multipole contribution. Both have a strong influence on spectral selectivity.

\section{ACKNOWLEDGMENT}

S. Schelm thanks BASF Aktiengesellschaft, Germany, for support of his $\mathrm{PhD}$ thesis.

Correspondence can be addressed to $S$. Schelm (stefan.schelm@gmail.com) or G. B. Smith (geoff.smith@uts.edu.au).

\section{REFERENCES}

1. C. Sönnichsen, T. Franzl, T. Wilk, G. von Plessen, J Feldmann, O. Wilson, and P. Mulvaney, "Drastic reduction of plasmon damping in gold nanorods," Phys. Rev. Lett. 88, 077402 (2002)

2. S. J. Oldenburg, R. D. Averitt, S. L. Westcott, and N. J. Halas, "Nanoengineering of optical resonances," Chem. Phys. Lett. 288, 243-247 (1998)

3. R. Baer, D. Neuhauser, and S. Weiss, "Enhanced absorption induced by a metallic nanoshell," Nano Lett. 4 85-88 (2004).

4. S. L. Westcott, J. B. Jackson, C. Radloff, and N. J. Halas, "Relative contributions to the plasmon line shape of metal nanoshells," Phys. Rev. B 66, 155431 (2002).

5. G. B. Smith and V. N. Pustovit, "Coupled multipolar interactions in clusters of nanoparticles with metal shells," Opt. Commun. 211, 197-204 (2002).

6. C. Graf and A. van Blaaderen, "Metallodielectric colloidal core-shell particles for photonic applications," Langmuir 18, 524-534 (2002)

7. S. J. Oldenburg, S. L. Westcott, R. D. Averitt, and N. J. Halas, "Surface enhanced Raman scattering in the near infrared using metal nanoshell substrates," J. Phys. Chem. B 111, 4729-4735 (1999).

8. A. J. Haes, S. L. Zou, G. C. Schatz, and R. P. Van Duyne, "A nanoscale optical biosensor: the long range distance dependence of the localized surface plasmon resonance of noble metal nanoparticles," J. Chem. Phys. 108, 109-116 (2004).

9. C. F. Bohren and D. R. Huffman, Absorption and Scattering of Light by Small Particles, Wiley Science Paperback Series (Wiley-Interscience, 1998).

10. U. Kreibig and M. Vollmer, Optical Properties of Metal Clusters, Springer Series in Materials Science (SpringerVerlag, 1995)

11. J. H. Weaver, C. Krafka, D. W. Lynch, and E. E. Koch, 
Optical Properties of Metals, Part II, Physics Data No. 18-2 (Fachinformationszentrum Energie, Physik, Mathematik, 1981).

12. E. D. Palik, Handbook of Optical Constants of Solids (Academic, 1985).

13. U. Kreibig and L. Genzel, "Optical absorption of small metallic particles," Surf. Sci. 156, 678-700 (1985).

14. B. N. J. Persson, "Polarizability of small spherical metal particles: influence of the matrix environment," Surf. Sci. 281, 153-162 (1993)

15. A. Pinchuk, U. Kreibig, and A. Hilger, "Optical properties of metaliic nanoparticles: infiuence of interface effects and interband transitions," Surf. Sci. 557, 269-280 (2004).

16. S. Schelm and G. B. Smith, "Dilute LaB6 nanoparticles in polymer as optimized clear solar control glazing," Appl. Phys. Lett. 82, 4346-4348 (2003).

17. S. Schelm, G. B. Smith, G. Wei, A. Vella, L. Wieczorek,
K.-H. Müller, and B. Raguse, "Double effective medium model for the optical properties of self-assembled gold nanoparticle films cross-linked with alkane dithiols," Nano Lett. 4, 335-339 (2004).

18. M. Brust, D. Bethell, C. J. Kiely, and D. J. Schiffrin, "Self-assembled gold nanoparticle thin films with nonmetallic optical and electronic properties," Langmuir 14, 5425-5429 (1998)

19. C. G. Granqvist and O. Hunderi, "Optical absorption of ultrafine metal spheres with dielectric cores," Z. Phys. B 30, 47-51 (1978).

20. R. D. Averitt, D. Sarkar, and N. J. Halas, "Plasmon resonance shifts of $\mathrm{Au}$-coated $\mathrm{Au}_{2} \mathrm{~S}$ nanoshells: insight into multicomponent nanoparticle growth," Phys. Rev. Lett. 78, 4217-4220 (1997)

21. R. D. Averitt, S. L. Westcott, and N. J. Halas, "Ultrafast electron dynamics in gold nanoshells," Phys. Rev. B 58, R10203-R10206 (1998). 